PROCEEDINGS OF THE AMERICAN MATHEMATICAL SOCIETY

Volume 126, Number 11, November 1998, Pages 3293-3298

S 0002-9939(98)04692-9

\title{
TRIANGULAR EXTENSION SPECTRUM OF WEIGHTED SHIFTS
}

\author{
ZHIDONG PAN
}

(Communicated by Palle E. T. Jorgensen)

\begin{abstract}
A necessary and sufficient condition for a complex number to be in the triangular extension spectrum of a weighted backward shift is obtained. It is shown that the triangular extension spectrum of a weighted backward shift is always a closed annulus when it is not empty. Moreover, for any given closed annulus, there exists a weighted backward shift with the annulus as its triangular extension spectrum.
\end{abstract}

A bounded operator $T$ acting on a separable Hilbert space $H$ is called triangular if there exists an orthonormal basis $\left\{e_{n}\right\}_{1}^{\infty}$ for $H$ such that $T e_{n}$ is in the span of $\left\{e_{1}, e_{2}, \ldots, e_{n}\right\}$ for each $n$. A vector $x$ is called an algebraic vector for an operator $T$ if there exists a nonzero polynomial $p(z)$ such that $p(T) x=0$. Let $\mathcal{E}_{T}$ be the set of algebraic vectors for an operator $T$. It can be shown that $T$ is triangular if and only if $\mathcal{E}_{T}$ is dense in $H . T$ is called algebraic if there exists a nonzero polynomial $p(z)$ so that $p(T)=0$. An operator $A$ is called semitriangular if $A$ is an extension of a triangular operator by a finite rank operator, or equivalently, the norm closure of the set of algebraic vectors has finite codimension in $H$. The finite codimension is called the index of semitriangularity of $A$. Semitriangular operators have been proved to be very useful in constructing counter-examples. Several longstanding open questions were answered negatively in [W], which led to the study of semitriangular operators; see [HLP], [HLW], [LW1], [LW2].

Let $B(H)$ be the set of all bounded linear operators on $H, \sigma(T)$ denote the spectrum of $T$ in $B(H), \sigma_{e}(T)$ denote the essential spectrum of $T$, and let $\mathbb{N}$ be the set of natural numbers. For any bounded sequence of complex numbers $\left\{s_{n}\right\}_{1}^{\infty}$, we define the corresponding weighted backward shift $S$ with respect to $\left\{e_{n}\right\}_{1}^{\infty}$ such that $S e_{1}=0, S e_{2}=s_{1} e_{1}, S e_{3}=s_{2} e_{2}, \ldots, S e_{i+1}=s_{i} e_{i}, \ldots$ Clearly, $\|S\|=\sup \left\{\left|s_{i}\right|\right\}$. Throughout this note, we always let $\mathbb{N}_{0}=\left\{i \in \mathbb{N}: s_{i}=0\right\}$.

The triangular extension spectrum was first introduced in [HLP] and it was shown that the triangular extension spectrum plays a very important role in studying semitriangular operators and algebraic extensions of triangular operators.

Definition 1. Let $T \in B(H)$ be a triangular operator. The triangular extension spectrum of $T$, denoted by $\sigma_{\Delta}(T)$, is defined to be the set of all $t \in \mathbb{C}$ such that

Received by the editors August 21, 1996 and, in revised form, February 28, 1997 and March 28, 1997.

1991 Mathematics Subject Classification. Primary 47A15, 47A45, 47C05.

Key words and phrases. Operator, triangular, semitriangular, extension, spectrum.

This work was supported in part by a research release time award from Saginaw Valley State University. 
there exists a $b \in B(\mathbb{C}, H)(=H)$ with the property that

$$
A=\left(\begin{array}{ll}
T & b \\
0 & t
\end{array}\right)
$$

has index of semitriangularity 1 in $H \oplus \mathbb{C}$. Let $\rho_{\Delta}(T)$ denote the complement of $\sigma_{\Delta}(T)$ in $\mathbb{C}$.

Lemma 2 ([HLP]). If $T$ is a triangular operator, then $t \in \sigma_{\Delta}(T)$ if and only if $\mathcal{E}_{T}+\operatorname{Ran}(T-t I) \neq H$.

It follows from the above lemma that $\sigma_{\Delta}(T) \subseteq \sigma(T)$. In fact, it is shown that $\sigma_{\Delta}(T)$ is always a compact subset of $\sigma_{e}(T)$ ([HLP]). Theorem 2.7 of [HLW] implies $\sigma_{\Delta}(T)=\varnothing$ if and only if $T$ is algebraic.

Lemma $3([\mathrm{HLW}])$. If $\mathcal{E}_{T}+\operatorname{Ran}(T-t I)=H$, then there exists a positive integer $k$ such that $\operatorname{ker}(T-t I)^{k}+\operatorname{Ran}(T-t I)=H$.

Lemma 4. Let $S$ be a weighted backward shift. If $S$ is not algebraic, then

$$
0 \in \sigma_{\Delta}(S) \text { if and only if } \underline{\lim _{i \rightarrow \infty}}\left|s_{i}\right|=0 .
$$

Proof. " $\Leftarrow "$ Case 1: $\mathbb{N}_{0}$ is infinite.

Suppose $\mathbb{N}_{0}=\left\{n_{1}, n_{2}, \ldots\right\}$ such that $n_{1}<n_{2}<n_{3}<\ldots$. It is easy to see that $S$ is algebraic if and only if $S$ is nilpotent if and only if $\mathbb{N}_{0}$ is infinite and $\sup \left\{n_{j+1}-n_{j}\right\}$ is finite. Let $H_{0}=\left[e_{n_{1}}, e_{n_{2}}, \ldots, e_{n_{i}}, \ldots\right]$, where [.] denotes the closed linear span. Since $S e_{n_{i}+1}=s_{n_{i}} e_{n_{i}}=0, \operatorname{Ran}(S) \subseteq H_{0}^{\perp}$. Since $S$ is not nilpotent, thus $\forall k \in \mathbb{N}, \exists m_{k}$ such that $S^{k} e_{m_{k}} \neq 0$. Suppose $n_{j_{k}-1}<m_{k} \leq n_{j_{k}}$; then $S^{k} e_{n_{j_{k}}} \neq 0$. Let $b=\sum_{i=1}^{\infty} \frac{1}{i} e_{n_{i}}$. We show that $b \notin \operatorname{ker}\left(S^{k}\right)+\operatorname{Ran}(S)$ for any $k$, which, by Lemma 2 and Lemma 3 , implies $0 \in \sigma_{\Delta}(S)$.

Suppose $b=u+S v$, where $u \in \operatorname{Ker}\left(S^{k}\right)$. Let $u=\sum_{i=1}^{\infty} u_{i} e_{i}$.

Since $\operatorname{Ran}(S) \subseteq H_{0}^{\perp}$, we have $u_{n_{i}}=\frac{1}{i}$.

Since $S^{k} u=\sum_{i=1}^{\infty} u_{i} S^{k} e_{i}=0$ and $\left\langle S^{k} e_{i}, S^{k} e_{j}\right\rangle=0$, for all $i \neq j$, where $\langle\cdot\rangle$ denotes the inner product, we have $u_{i} S^{k} e_{i}=0$, for all $i$, in particular, $u_{n_{i}} S^{k} e_{n_{i}}=0$, i.e. $\frac{1}{i} S^{k} e_{n_{i}}=0$, thus $S^{k} e_{n_{i}}=0$, for all $i$, which is a contradiction.

Case 2: $\mathbb{N}_{0}$ is finite.

Let $P_{n}$ be the orthogonal projections of $H$ onto $\left[e_{1}, e_{2}, \ldots, e_{n}\right]$ for $n=1,2,3, \ldots$. We need to show that $\operatorname{ker}\left(S^{k}\right)+\operatorname{Ran}(S) \neq H$. Since $\operatorname{ker}\left(S^{i}\right) \subseteq \operatorname{ker}\left(S^{i+1}\right)$ for all $i$, we only need to show that $\operatorname{ker}\left(S^{k}\right)+\operatorname{Ran}(S) \neq H$ for $k$ large enough. Without loss of generality, we assume $k$ is large enough so that $s_{i} \neq 0$ for all $i>k$. For any $x=\sum_{i=1}^{\infty} x_{i} e_{i} \in \operatorname{ker}(S), S x=\sum_{i=1}^{\infty} x_{i} s_{i} e_{i}=0$. Since $\mathbb{N}_{0}$ is finite, we have all but finitely many $x_{i}$ 's are zero. Therefore there exists an $N$ such that $\operatorname{ker}(S) \subseteq P_{N} H$, so $\operatorname{ker}\left(S^{k}\right) \subseteq P_{M} H$, where $M=N+k$. If $\operatorname{ker}\left(S^{k}\right)+\operatorname{Ran}(S)=H$, then $\operatorname{Ran}\left(P_{M}^{\perp} S\right)=$ $P_{M}^{\perp} \operatorname{Ran}(S)=P_{M}^{\perp} H$. Note that $P_{M}^{\perp} S=P_{M}^{\perp} S P_{M+1}^{\perp}$ and $s_{i} \neq 0$, for $i>M$. Hence $P_{M}^{\perp} S P_{M+1}^{\perp}$ is a $1-1$ and onto map from $P_{M+1}^{\perp} H$ to $P_{M}^{\perp} H$. Therefore $P_{M}^{\perp} S P_{M+1}^{\perp}$ is invertible by the open mapping theorem, but since $\underline{\lim }\left|s_{i}\right|=0$, there exists a subsequence $s_{n_{j}}$ such that $s_{n_{j}} \rightarrow 0$ as $j \rightarrow \infty$. Thus $P_{M}^{\perp} S P_{M+1}^{\perp} e_{n_{j}+1}=s_{n_{j}} e_{n_{j}} \rightarrow 0$ as $j \rightarrow \infty$, contradicting the fact that $P_{M}^{\perp} S P_{M+1}^{\perp}$ is invertible.

" $\Rightarrow$ " If $\underline{\lim }_{i \rightarrow \infty}\left|S_{i}\right| \neq 0$, then there exist an $\varepsilon_{0}>0$ and a $k_{0} \in \mathbb{N}$ such that $\left|s_{i}\right|>\varepsilon_{0}$, for all $i>k_{0}$. For any $x \in P_{k_{0}}^{\perp} H, x=\sum_{i=k_{0}+1}^{\infty} x_{i} e_{i}$, let $y=\sum_{i=k_{0}+1}^{\infty} \frac{1}{s_{i}} x_{i} e_{i+1}$; then $S y=x$, i.e. $\operatorname{Ran}(S) \supseteq P_{k_{0}}^{\perp} H$. Since $P_{k_{0}} H \subseteq \operatorname{ker}\left(S^{k}\right)$ for $k$ large enough, we have 
$\operatorname{ker}\left(S^{k}\right)+\operatorname{Ran}(S)=H$ for $k$ large enough. Therefore, by Lemma 2 and Lemma 3, $0 \notin \sigma_{\Delta}(S)$.

Theorem 5. Let $S$ be a weighted backward shift. If $\mathbb{N}_{0}$ is infinite and $S$ is not algebraic, then $\sigma_{\Delta}(S)=\sigma(S)$.

Proof. We only need to show that $\sigma(S) \subseteq \sigma_{\Delta}(S)$. Let $t \in \sigma(S)$. If $t=0$, then $t \in \sigma_{\Delta}(S)$ by Lemma 4. If $t \neq 0$, then $\operatorname{ker}(S-t I)=0$. By Lemma 2 and Lemma $3, t \in \sigma_{\Delta}(S)$ if and only if $\operatorname{Ran}(S-t I) \neq H$, which is equivalent to $t \in \sigma(S)$ by the open mapping theorem, since $\operatorname{ker}(S-t I)=0$.

Lemma 6 ([S]). If $S$ is a weighted backward shift with spectral radius $r$, then $\sigma(S)=\{t:|t| \leq r\}$.

The following is essentially Theorem 8 of $[\mathrm{S}]$.

Lemma 7. Let $S$ be a weighted backward shift. Suppose that $\mathbb{N}_{0}$ is finite with $k_{0}=\max \left\{i: i \in \mathbb{N}_{0}\right\}$. If $t \neq 0$, then $S-t I$ is not $1-1$ if and only if

$$
\sum_{n=k_{0}+1}^{\infty}\left|\frac{t^{n-k_{0}}}{s_{n} \cdots s_{k_{0}+1}}\right|^{2}<\infty
$$

In this case, $\operatorname{dim} \operatorname{ker}(S-t I)=1$.

Lemma 8. If $t \in \sigma(S)$ and $t \neq 0$, then $t \in \rho_{\Delta}(S) \Leftrightarrow \operatorname{Ran}(S-t I)=H$.

Proof. From Lemma $7, \operatorname{dim} \operatorname{ker}(S-t I)$ is at most one, so for any $k, \operatorname{dim} \operatorname{ker}(S-t I)^{k}$ is finite (in fact at most $k$ ). For any natural number $n$ and $y \in\left[e_{1}, \ldots, e_{n}\right]$, we can solve $(S-t I) x=y$ for $x$. Thus $\operatorname{Ran}(S-t I)$ is dense in $H$. Combining the above with Lemma 2 and Lemma 3, we have $t \in \rho_{\Delta}(S) \Leftrightarrow \exists k, \ni \operatorname{ker}(S-t I)^{k}+\operatorname{Ran}(S-t I)=$ $H \Leftrightarrow \operatorname{Ran}(S-t I)=H$.

Corollary 9. If $S$ is a weighted backward shift and $S$ is not algebraic, then $\sigma_{\Delta}(S)=$ $\sigma_{e}(S)$.

Proof. This follows immediately from Lemma 4 and Lemma 8.

Lemma 10 ([HLP]). Suppose that $A \in B(H)$ has a closed range and let $P$ be the orthogonal projection of $H$ onto $\operatorname{Ran}(A)$. Then there exists an $\varepsilon>0$ such that $\operatorname{Ran}(A) \subseteq \operatorname{Ran}(A+\lambda P)$ for all $\lambda$ with $|\lambda|<\varepsilon$.

Corollary 11. If $\operatorname{Ran}(S-t I)=H$, then there exists an $\varepsilon>0$ such that $\operatorname{Ran}(S-z I)=H$ for all $z$ with $|z-t|<\varepsilon$.

Lemma 12. Suppose that $\mathbb{N}_{0}$ is finite with $k_{0}=\max \left\{i: i \in \mathbb{N}_{0}\right\}$. If $t \in \sigma(S)$ and $t \neq 0$, then $t \in \rho_{\Delta}(S)$ if and only if there exist an $M$ and $a z_{0}$ with $\left|z_{0}\right|>|t|$ such that

$$
\left|\frac{z_{0}^{n}}{s_{k+n} s_{k+n-1} \cdots s_{k}}\right| \leq M, \quad \forall k>k_{0}, n>0 .
$$

Proof. " $\Rightarrow$ " If $t \in \rho_{\Delta}(S)$, and $t \neq 0$, then by Lemma $8, \operatorname{Ran}(S-t I)=H$. Since $t \in \sigma(S)$ and $S-t I$ is surjective, $t \notin \partial \sigma(S)$. Combining with Corollary 11, we can find a disk (centered at $t$ ) contained in $\sigma(S)$ with the property that $\operatorname{Ran}(S-z I)=H$ for all $z$ in the disk. Since the disk is contained in the spectrum, we have $S-z I$ is 
not 1-1 for all $z$ in the disk. In particular, there exists a $z_{0}$ with $\left|z_{0}\right|>|t|$ so that $\operatorname{Ran}\left(S-z_{0} I\right)=H$ and $S-z_{0} I$ is not 1-1. By Lemma 7 ,

$$
\sum_{n=0}^{\infty} \frac{\left|z_{0}^{2 n}\right|}{\left|s_{k_{0}+n+1} \cdots s_{k_{0}+1}\right|^{2}}<\infty
$$

Hence $S-z_{0} I$ is onto and 1-1 from $\operatorname{ker}\left(S-z_{0} I\right)^{\perp}$ to $H$, so it has a bounded inverse $B$ from $H$ to $\operatorname{ker}\left(S-z_{0} I\right)^{\perp}$.

For any $k>k_{0}$, let $x^{(k)}=B e_{k}$, write $x^{(k)}=\sum_{i=1}^{\infty} x_{i}^{(k)} e_{i}$; then $\left\|x^{(k)}\right\| \leq\|B\|$, in particular, $\left|x_{i}^{(k)}\right| \leq\|B\|$ for all $i$. Let

$$
M=\left\{1+\left(\sum_{n=0}^{\infty} \frac{\left|z_{0}^{2 n}\right|}{\left|s_{k_{0}+n+1} \cdots s_{k_{0}+1}\right|^{2}}\right)^{1 / 2}\right\}\|B\| .
$$

Since $\left(S-z_{0} I\right) x^{(k)}=\left(S-z_{0} I\right) B e_{k}=e_{k}$, we have

$$
s_{i} x_{i+1}^{(k)}-z_{0} x_{i}^{(k)}=0, \quad i \neq k, \quad \text { and } \quad s_{k} x_{k+1}^{(k)}-z_{0} x_{k}^{(k)}=1 .
$$

From the above, we can obtain

$$
x_{k}^{(k)}=\frac{z_{0}}{s_{k-1}} x_{k-1}^{(k)}=\cdots=\frac{z_{0}^{k-k_{0}-1}}{s_{k-1} \cdots s_{k_{0}+1}} x_{k_{0}+1}^{(k)} .
$$

Now for any positive integer $n$,

$$
\begin{aligned}
x_{k+n+1}^{(k)} & =\frac{z_{0}}{s_{k+n}} x_{k+n}^{(k)}=\cdots=\frac{z_{0}^{n}}{s_{k+n} \cdots s_{k+1}} x_{k+1}^{(k)} \\
& =\frac{z_{0}^{n}}{s_{k+n} \cdots s_{k+1}}\left(\frac{z_{0}}{s_{k}} x_{k}^{(k)}+\frac{1}{s_{k}}\right)=\frac{z_{0}^{n}}{s_{k+n} \cdots s_{k}} x_{k}^{(k)}+\frac{z_{0}^{n}}{s_{k+n} \cdots s_{k}} \\
& =\frac{z_{0}^{n}}{s_{k+n} \cdots s_{k}}\left(\frac{z_{0}^{k-k_{0}-1}}{s_{k-1} \cdots s_{k_{0}+1}} x_{k_{0}+1}^{(k)}\right)+\frac{z_{0}^{n}}{s_{k+n} \cdots s_{k}} \\
& =\frac{z_{0}^{n+k-k_{0}-1}}{s_{k+n} \cdots s_{k_{0}+1}} x_{k_{0}+1}^{(k)}+\frac{z_{0}^{n}}{s_{k+n} \cdots s_{k}} .
\end{aligned}
$$

Thus

$$
\frac{z_{0}^{n}}{s_{k+n} \cdots s_{k}}=x_{k+n+1}^{(k)}-\frac{z_{0}^{n+k-k_{0}-1}}{s_{k+n} \cdots s_{k_{0}+1}} x_{k_{0}+1}^{(k)}
$$

Therefore

$$
\left|\frac{z_{0}^{n}}{s_{k+n} \cdots s_{k}}\right| \leq\left|x_{k+n+1}^{(k)}\right|+\left|\frac{z_{0}^{n+k-k_{0}-1}}{s_{k+n} \cdots s_{k_{0}+1}}\right|\left|x_{k_{0}+1}^{(k)}\right| \leq M .
$$

" $\Leftarrow$ " Note that when $n=1,(* *)$ implies $\left|\frac{z_{0}}{s_{k+1} s_{k}}\right| \leq M, \forall k>k_{0}$, so

$$
\left|\frac{1}{s_{k}}\right| \leq M\left|\frac{s_{k+1}}{z_{0}}\right| \leq M \frac{\|S\|}{\left|z_{0}\right|}, \quad \forall k>k_{0} .
$$

Let $H_{1}=\left[e_{k_{0}+1}, e_{k_{0}+2}, \ldots\right]$. We only need to show $\operatorname{Ran}(S-t I) \supseteq H_{1}$.

First, we define a bounded linear operator $R$ on $H_{1}$ such that $R e_{i}=\frac{1}{s_{i}} e_{i+1}, \forall i \geq$ $k_{0}+1$. It follows that $\|R\| \leq M$ and $S R=I_{H_{1}}$. Since $\left(z_{0} R\right)^{n} e_{i}=\frac{z_{0}^{n}}{s_{i+n-1} \cdots s_{i}} e_{i+n}$, we have $\left\|\left(z_{0} R\right)^{n}\right\| \leq \sup \left\{\left|\frac{z_{0}^{n}}{s_{i+n-1} \cdots s_{i}}\right|: i \geq k_{0}+1\right\} \leq M\left|z_{0}\right|$, which implies $\left\|(t R)^{n}\right\|=$ 
$\left|\frac{t}{z_{0}}\right|^{n}\left\|\left(z_{0} R\right)^{n}\right\| \leq\left|\frac{t}{z_{0}}\right|^{n} M\left|z_{0}\right|$. Therefore, $Q \equiv \sum_{n=1}^{\infty}(t R)^{n}$ defines a bounded linear operator on $H_{1}$. Given any $y \in H_{1}$, let $x=Q y$; then

$$
\begin{aligned}
(S-t I) x & =(S-t I) Q y=(S-t I) \sum_{n=1}^{\infty}(t R)^{n} y \\
& =\left(S \sum_{n=1}^{\infty}(t R)^{n}-t \sum_{n=1}^{\infty}(t R)^{n}\right) y \\
& =\left(S t R+S \sum_{n=2}^{\infty}(t R)^{n}-t \sum_{n=1}^{\infty}(t R)^{n}\right) y \\
& =\left(t I_{H_{1}}+t I_{H_{1}} \sum_{n=1}^{\infty}(t R)^{n}-t \sum_{n=1}^{\infty}(t R)^{n}\right) y=t y .
\end{aligned}
$$

This shows $\operatorname{Ran}(S-t I) \supseteq H_{1}$.

Remark. (i) (**) implies $(*)$. This is because if $(* *)$ holds, then

$$
\begin{aligned}
\sum_{n=0}^{\infty} \frac{\left|t^{2 n}\right|}{\left|s_{k_{0}+n+1} \cdots s_{k_{0}+1}\right|^{2}} & =\sum_{n=0}^{\infty}\left(\left|\frac{t}{z_{0}}\right|^{2 n} \frac{\left|z_{0}^{2 n}\right|}{\left|s_{k+0+n+1} \cdots s_{k_{0}+1}\right|^{2}}\right) \\
& \leq \sum_{n=0}^{\infty}\left(\left|\frac{t}{z_{0}}\right|^{2 n} M^{2}\right)<\infty .
\end{aligned}
$$

(ii) $(* *)$ is equivalent to the following: For any fixed $N>k_{0}$, there exist an $M$ and a $z_{0}$ with $\left|z_{0}\right|>|t|$ such that

$$
\left|\frac{z_{0}^{n}}{s_{k+n} s_{k+n-1} \cdots s_{k}}\right| \leq M, \quad \forall k>N, n>0 .
$$

Similarly, $(*)$ is equivalent to: For any $N>k_{0}$,

$$
\sum_{n=N}^{\infty}\left|\frac{t^{n-N+1}}{s_{n} \cdots s_{N}}\right|^{2}<\infty .
$$

(iii) In the proof of Lemma 12, we see that (**) implies $\left|\frac{1}{s_{i}}\right| \leq M \frac{\|S\|}{\left|z_{0}\right|}, \forall i>k_{0}$, i.e. $\lim _{i \rightarrow \infty}\left|s_{i}\right| \neq 0$.

If $\mathbb{N}_{0}$ is finite, we define $r_{1} \equiv \inf \{|t|: t$ does not satisfy $(* *)\}, r_{2} \equiv \inf \{|t|: t$ does not satisfy $(*)\}$. (In the case $\mathbb{N}_{0}$ is infinite, we define $r_{1}=r_{2}=0$.)

Since $(* *)$ implies $(*)$, we have $r_{1} \leq r_{2}$.

Theorem 13. If $S$ is a weighted backward shift and $S$ is not algebraic, then $\sigma_{\Delta}(S)$ $=\left\{t: r_{1} \leq|t| \leq r\right\}$. In particular, if $S$ is the standard backward shift, then $\sigma_{\Delta}(S)=$ $\{t:|t|=1\}$.

Proof. This follows directly from Theorem 5, Lemma 6 and Lemma 12.

Lemma 12 together with Lemma 7 implies the next corollary which gives a more descriptive picture of the spectrum of a weighted backward shift.

Corollary 14. With assumptions as above, we have the following:

(i) If $|t|<r_{1}$, then $S-t I$ is onto but not 1-1.

(ii) If $r_{1} \leq|t|<r_{2}$, then $S-t I$ is neither 1-1 nor onto. 
(iii) If $r_{2}<|t| \leq r$, then $S-t I$ is 1-1 but not onto.

(iv) If $|t|<r$, then $S-t I$ is invertible.

Remark. $S-r_{2} I$ can be 1-1 in some cases and not 1-1 in others. In the case $r_{1}=r_{2}$, we do not have (ii) of the above theorem and in the case $r_{2}=r$, we do not have (iii) of the above theorem. However, we always have if $r_{1} \leq|t| \leq r$, then $S-t I$ is not onto, that is, $\sigma_{\Delta}(S)=\left\{t: r_{1} \leq|t| \leq r\right\}$ if $S$ is not algebraic.

The following example shows we can alway choose a weighted backward shift with desired $r_{1}, r_{2}$ and $r$. For simplicity, we assume $r=1$.

Example. Suppose that $0<a<b<1$. Defined a weighted backward shift as follows: For $k=0,1,2 \ldots$, define

$$
\begin{aligned}
& s_{i}=1 \quad \frac{1}{2} k(k+1)(n+2)<i \leq \frac{1}{2} k(k+1)(n+2)+(k+1), \\
& s_{i}=a \quad \frac{1}{2} k(k+1)(n+2)+(k+1)<i \leq \frac{1}{2} k(k+1)(n+2)+2(k+1), \\
& s_{i}=d \quad \frac{1}{2} k(k+1)(n+2)+2(k+1)<i \leq \frac{1}{2} k(k+1)(n+2)+(n+2)(k+1)
\end{aligned}
$$

where $n$ is fixed such that $\left(\frac{a}{b}\right)^{n+1} \leq b$ and $b^{n+1} \leq \frac{a}{b}$, and $d=\sqrt[n]{\frac{b^{n+2}}{a}}$. It can be verified that $r_{1}=a, r_{2}=b$ and $r=1$.

Corollary 15. For any $L_{1}<L_{2}$, there exists a weighted backward shift $S$ such that $\sigma_{\Delta}(S)=\left\{t: L_{1} \leq|t| \leq L_{2}\right\}$.

The author would like to thank Professor David Larson for valuable suggestions. The author would also like to thank the referee for several very helpful suggestions and for pointing out an error in the original proof of Lemma 12.

\section{REFERENCES}

[HLP] D. Han, D. Larson and Z. Pan, The triangular extension spectrum and algebraic extensions of operators, preprint.

[HLW] D. Herrero, D. Larson and W. Wogen, Semitriangular operators, Houston J. Math. 17 (1991), 477-499. MR 92m:47037

[LW1] D. Larson and W. Wogen, Extensions of bitriangular operators, Integ. Eq. and Oper. Th. 25 (1996), 216-223. MR 97d:47027

[LW2] _ Some problems on triangular and semitriangular operators, Contemporary Mathematics 120 (1991), 97-100. MR 92i:47019

[LW3] _ Reflexivity of $T \oplus 0$, J. Funct. Anal. 92 (1990), 448-467. MR 91i:47010

[LW4] _ Extension of normal operators, Integ. Eq. and Oper. Th. 20 (1994), 325-334. MR 96b: 47024

[S] A. Shields, Weighted shift operators and analytic function theory, Topics in Operator Theory, Math Surveys, no. 13, Amer. Math. Soc., Providence, R.I., 1974, pp. 49-128. MR 50:14341

[W] W. Wogen, Some counterexamples in non-self-adjoint algebras, Ann. of Math. 126 (1987), 415-427. MR 89b:47066

Department of Mathematics, Saginaw Valley State University, University Center, Michigan 48710

E-mail address: pan@tardis.svsu.edu 